\title{
ARTICLE
}

\section{Progress and Applications of MCAM: Monte Carlo Automatic Modeling Program for Particle Transport Simulation}

\author{
Guozhong WANG ${ }^{1,2, *}$, Jian XIONG ${ }^{1,2}$, Pengcheng LONG $^{1,2}$, Dianxi WANG ${ }^{1,2}$, Kai ZHAO $^{1,2}$, \\ Qin ZENG ${ }^{1,2}$, Liqin HU ${ }^{1,2}$, Dongchuan YING ${ }^{1,2}$, Junjun ZHANG ${ }^{1,2}$, Akio SAGARA ${ }^{3}$, \\ Teruya TANAKA ${ }^{3}$ and Takeo MUROGA ${ }^{3}$ \\ ${ }^{1}$ University of Science and Technology of China, P.O. Box 6022, Hefei, Anhui, 230029, China \\ ${ }^{2}$ Institute of Plasma Physics, Chinese Academy of Sciences, P.O. Box 1126, Hefei, Anhui, 230031, China \\ ${ }^{3}$ National Institute for Fusion Science, 322-6, Oroshi, Toki, Gifu, 509-5292, Japan
}

\begin{abstract}
MCAM (Monte Carlo Automatic Modeling program for particle transport simulation) was developed by FDS Team as a bi-directional interface program between general CAD systems and Monte Carlo particle transport simulation codes. The physics and material modeling and void space modeling functions were improved and the free-form surfaces processing function was developed recently. The applications to the ITER (International Thermonuclear Experimental Reactor) building model and FFHR (Force Free Helical Reactor) model have demonstrated the feasibility, effectiveness and maturity of MCAM latest version for nuclear applications with complex geometry.
\end{abstract}

KEYWORDS: MCAM, Monte Carlo modeling, MCNP, void modeling, free-form surface

\section{Introduction}

Monte Carlo (MC) particle transport codes (e.g. MCNP, ${ }^{1)}$ TRIPOLI $^{2)}$ ) are widely used in the nuclear analysis. However, a bi-directional conversion interface program is needed to enhance the Monte Carlo modeling of nuclear devices with complex geometry. ${ }^{3)}$

MCAM $^{4-7)}$ (Monte Carlo Automatic Modeling Program for Particle Transport Simulation) was developed by FDS Team, China, as a bi-directional interface program between general commercial CAD (Computer Aided Design) systems and $\mathrm{MC}$ particle transport codes. On one hand, the engineering model created by CAD systems can be converted into the input geometry suitable for the MC codes conveniently and rapidly. On the other hand, the existing MC model can be inverted into CAD model and visualized for verifying and further updating. MCAM also supports a series of supplementary functions such as creation and repair of CAD models and analysis of physics properties.

The development of MCAM was started in 1999. More than 100 person-years have been invested. MCAM version 4.2 was a mature and efficient version which was benchmarked with ITER (International Thermonuclear Experimental Reactor) benchmark model. ${ }^{5)}$ MCAM version 4.7 was released in 2008 and has already been applied to many complex nuclear facilities successfully, including ITER ${ }^{8)}$ the superconducting tokamak being operated in China EAST, ${ }^{9)}$ FDS series reactors design ${ }^{10)}$ and Compact Reversed Shear Tokamak Reactor ${ }^{11)}$ (CREST). At present, MCAM can treat two Monte Carlo codes: MCNP and TRIPOLI.

*Corresponding author, E-mail:mango33@mail.ustc.edu.cn

(C) 2011 Atomic Energy Society of Japan, All Rights Reserved.
In the following, the recent achievements (physics and material modeling, void space modeling, free-form surface processing) and applications of MCAM to the ITER building model and FFHR (Force Free Helical Reactor) model are presented.

\section{New Capabilities}

\section{Physics and Material Modeling}

MCAM can now generate a full-formed input file of Monte Carlo codes from a CAD format file. Not only the geometry description can be generated automatically, but also the material, source, tally and other physics properties which Monte Carlo simulation need can be edited and assigned graphically. Therefore, no manually operations in text format are needed before a Monte Carlo simulation is carried out.

It is difficult to manually describe material information and assign it to correct cells in the modeling of Monte Carlo simulation. The material process function of MCAM was developed to edit the material description graphically. Different materials including mixture, compound and elements are saved as a database and displayed in list boxes. The material card for Monte Carlo simulation can be generated automatically after choosing the volume, mass or atom fraction of different materials from the list boxes. The material card includes the nuclide density, nuclide density percentage, mass density, and mass density percentage. Additionally, new material can be edited and added into the database and the material out of use can be deleted from the database. The User Interface is shown in Fig. 1. 


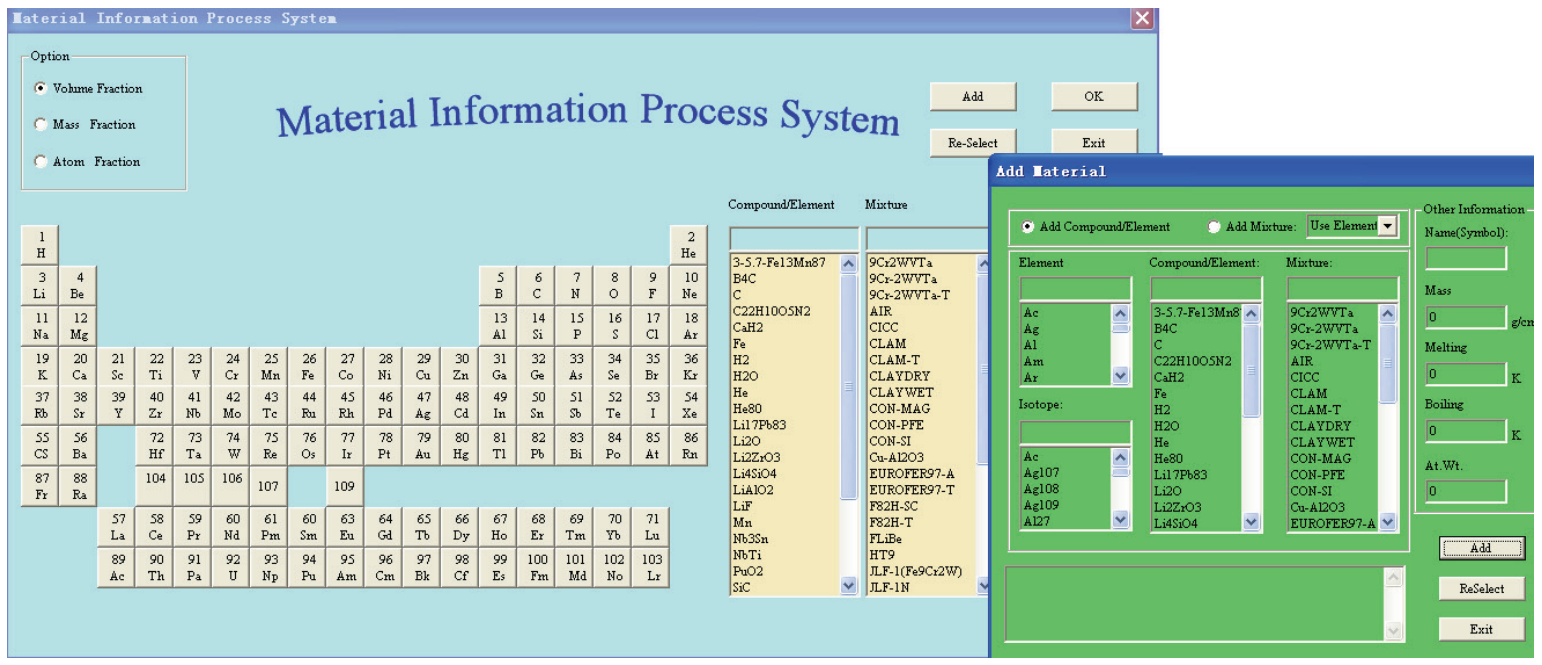

Fig. 1 The user interface of material process function

\section{Improved Void Space Modeling}

The commercial CAD systems only define the components of the engineering model (the solid region) generally, while it is needed to describe the void space (the vacuum region) in Monte Carlo simulation. At its simplest, the void space can be defined by boolean subtracting all the solid components from an encompassing volume. However, this method can introduce several problems. For example, the void model must be created using boolean operation whose, however, success rate cannot be ensured. Additionally, the created void model may be irregular and contain small surfaces or sharp corners.

Void space filling function of MCAM was improved to generate the void description accurately and efficiently. The whole void space was divided into several small cells. Each of the void cells was described as a cube subtracting the solid bodies interfacing with it using the "complement operator" in Monte Carlo codes (e.g., "\#” symbol in MCNP code). A big cube was firstly created, and then divided into small ones using the BSP (Binary Space Partitioning) method according to the geometry complexity of solid bodies interfacing with each void cell. The greatest advantage of this void space modeling method is that the boolean subtraction operations are transferred to the Monte Carlo codes and therefore the flaws and inconsistencies can be avoided. The use of BSP method could reduce the complexity of the void space cells because the partition of space was not symmetrical. The void cube could be bigger in the region where the solids are simpler and be smaller where the solids are more complex.

\section{Free-Form Surface Processing}

Free-form surfaces like non-uniform rational B-splines (NURBS) are widely used in the field of mechanical design and CAD systems as well as the analytical surfaces. However, they are not supported by Monte Carlo codes. Therefore, it is necessary to perform the process from the free form surfaces in CAD systems to the analytical surfaces supported by Monte Carlo codes.

Three methods for processing free form surfaces were developed according to the geometry features of free form surfaces. The Free Form Surface Approximating Method used a series of plane facets to approximate the free form surface. The Quadric Surface Fitting Method focuses on the quadratic surfaces (ellipsoid, paraboloid and hyperboloid surfaces) which are defined using free form surfaces in CAD systems. A general quadric surface equation was constructed to fit the sample points on the free form surface and then the type and parameters of quadric surface were parsed and identified corresponding to the definition in the Monte Carlo codes. The Swept Spline Surface Fitting Method focuses on the free form surfaces created by sweep operations in CAD systems. On the basis of discreting and approximating or fitting the cross section curve or/and the path curve of the swept spline surface, the new surface was recreated according to its constructive way.

Free form surface processing function with three code modules were developed on the basis of study of the methods. It achieved the accurate simulation of complex free form surfaces with planes, quadric surfaces and quartic surfaces supported by the Monte Carlo codes.

\section{Applications}

\section{ITER Building Model}

ITER organization has distributed the latest detailed three dimensional reference neutronics calculation model of tokamak complex. The model was successfully used in the radiation nuclear analysis of ITER tokamak complex. However, it extends only to the bio-shield. A detailed neutronics reference model of ITER building complex should be created and distributed to anticipate further neutronics analysis outside the bio-shield.

The ITER building consists of the tokamak building, the tritium building and the diagnostic building, which is $118 \mathrm{~m}^{*} 81 \mathrm{~m} * 73 \mathrm{~m}$ in size. The CAD model was created by 


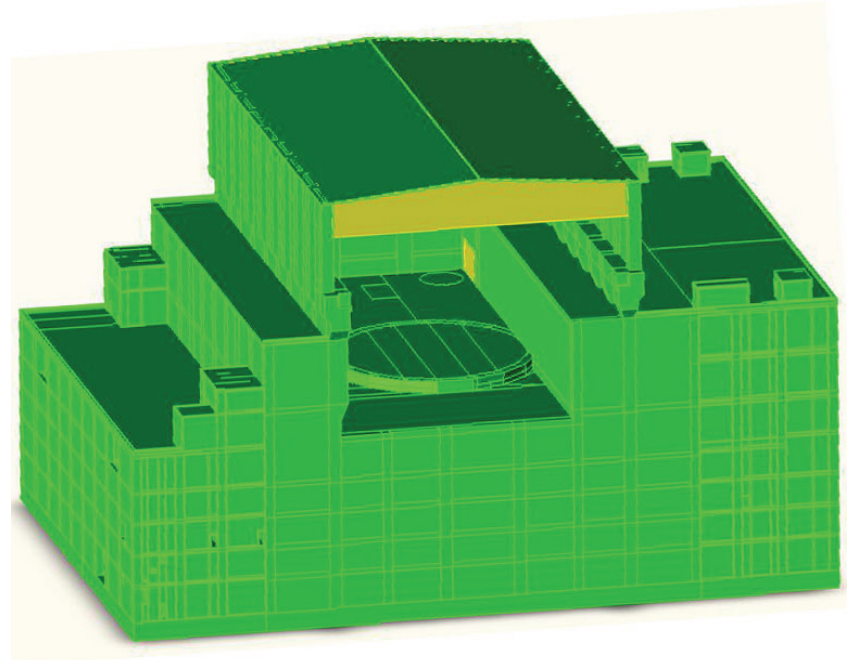

Fig. 2 The ITER Building model in MCAM

ITER organization using the CATIA software, which is a multi-platform commercial CAD software developed by the French company Dassault Systemes. The model was saved as the STEP (standard for the exchange of product model data) format.

With the help of MCAM, the neutronics model of ITER building was created successfully with high efficiency and accuracy. The model will be distributed as the reference model of ITER for the radiation analysis outside the bio-shield.

To approach the final neutronics model, the following processes were made with MCAM.

- Format conversion: the STEP format CAD model was imported into MCAM (see Fig. 2).

- Simplification: removing irrelevant details such as chamfers and screw threads.

- Modification: modifying the walkways, ladders and tokamak roof.

- Overlaps clearance.

- Physics and material modeling.

- The conversion from CAD model to the MCNP input file, together with the void space modeling.

By these processes, a complete MCNP input file of ITER building was produced, including the geometry description, the void space description, the material assignment, the source and tally definition and other physics properties.

For verification, the volumes and shapes of cells in MCNP model were compared and agreed very well with the original CAD model by means of stochastic volume calcula-

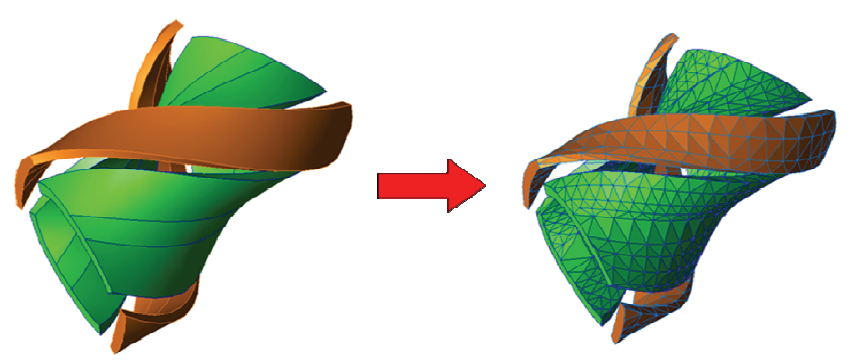

Fig. 3 Comparison of the blanket components before and after free form surfaces processing

tions and plot function which are supported by MCNP.

\section{FFHR Model}

MCAM was applied to the neutronics modeling of the large scale fusion reactor of stellarator FFHR (Force Free Helical Reactor) developed in NIFS (National Institute of Fusion Science), Japan, whose kernel components are mainly composed of complex free form surfaces.

The CAD model version was FFHR2m1. ${ }^{12)}$ It was created by NIFS with the three dimensional commercial CAD modeling software Pro/Engineering. As the model changed in a periodic of 36 degree in the toroidal direction, only a 36 degree sector model was adopted. The model includes the main components such as the breeder blanket, shielding blanket, helical coil, poloidal coil, cryostat, support structure. The helical structure of the blankets and coils was established with free form surfaces.

The "Free Form Surface Approximating" code module was used to process free form surfaces in the FFHR CAD model. The shape comparison before and after the process of the blanket components are shown in Fig. 3. The volume of some components was also compared, with a tolerance of less than $6 \%$, see Table 1 . The results show a good agreement between the model before and after processing in the shape and volume.

After the free form surfaces processing, then the new FFHR CAD model which do not contain free form surfaces was converted into MCNP simulation geometry model using MCAM. The cross sector view of FFHR geometry model in MCNP is shown in Fig. 4. Since the model was 36 degree sector, the periodic boundary function of the MCNP code was used.

The neutronics calculations were performed using MCNP-4C code and nuclear data library FENDL/MC-2.1. ${ }^{13}$ Tritium breeding ratios (TBRs) for the Flibe+Be/JLF-1 blanket systems were simulated. The material composition

Table 1 Volume comparison of the CAD model and the MCNP mode of the components containing free form surfaces

\begin{tabular}{lccccc}
\hline Component & $\begin{array}{c}\text { Original CAD } \\
\text { Model }\left(\mathrm{m}^{3}\right)\end{array}$ & $\begin{array}{c}\text { FDS CAD Model and } \\
\text { MCNP model }\left(\mathrm{m}^{3}\right)\end{array}$ & $\begin{array}{c}\text { Difference from } \\
\text { Original CAD model }\end{array}$ & $\begin{array}{c}\text { NIFS MCNP } \\
\text { Model }\left(\mathrm{m}^{3}\right)\end{array}$ & $\begin{array}{c}\text { Difference from } \\
\text { Original CAD model }\end{array}$ \\
\hline Breeder Blanket A & 19.85 & 18.00 & $-5.56 \%$ & 22.89 & $13.82 \%$ \\
Breeder Blanket B & 32.60 & 30.78 & $-5.58 \%$ & 36.98 & $12.50 \%$ \\
Shield A & 59.27 & 58.44 & $-1.40 \%$ & 73.89 & $24.67 \%$ \\
Shield B & 82.26 & 83.02 & $0.92 \%$ & 85.47 & $3.90 \%$ \\
Helical Coil & 65.91 & 64.07 & $-2.79 \%$ & 60.33 & $-8.47 \%$ \\
\hline
\end{tabular}




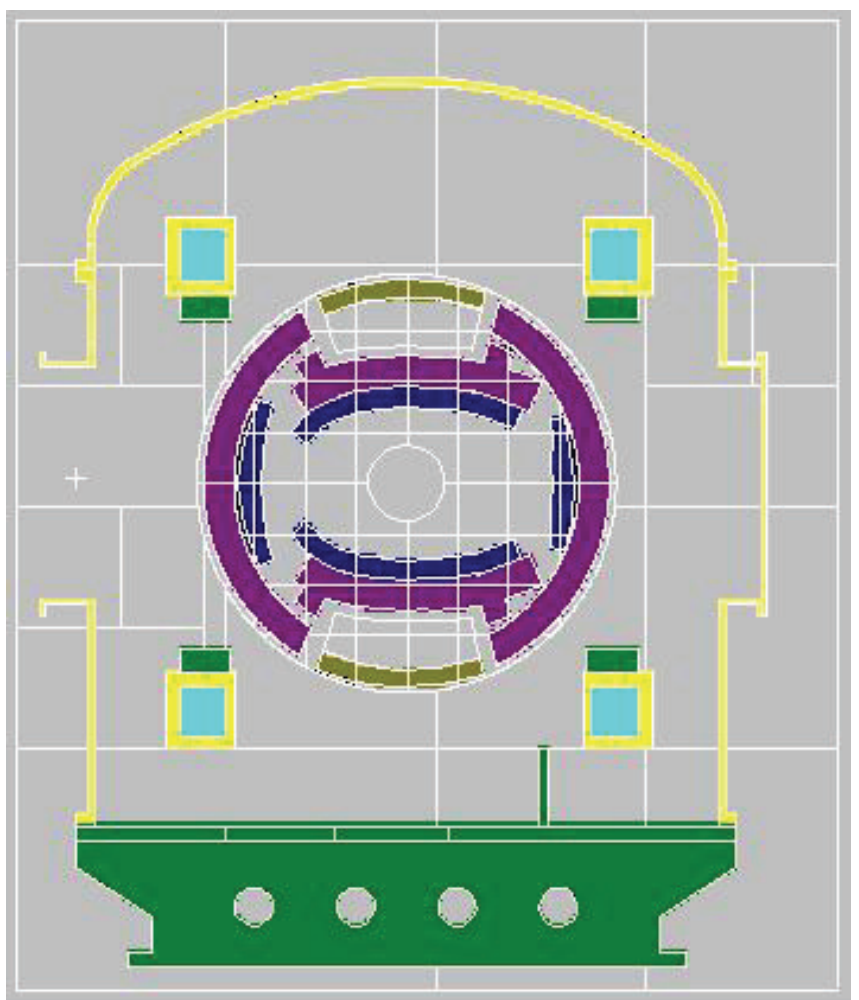

Fig. 4 Cross sector view of FFHR model created by MCAM in MCNP code

and neutron source were the same as those mentioned in Reference 14. The total TBRs were 0.94, which were lower compared with the previous results 1.08 in NIFS. ${ }^{14)}$ The difference was resulted from the difference of the MCNP model created by FDS Team and NIFS (see Table 1). The MCNP model created by NIFS was expanded in breed blanket and shield blanket in order to enhance the tritium breeding capability of the blanket design and was therefore larger than the original CAD model. ${ }^{14)}$ The MCNP model created by FDS was a little smaller in blanket than the original CAD model. The calculation result shows that the original CAD model does not satisfy the designed TBRs and therefore the design should be improved. The breeder and the shielding layers should be expanded, as which have been done in the neutronics analysis in Reference 14.

\section{Conclusions}

As an automatic modeling program for Monte Carlo particle transport simulation, MCAM implemented the bi-directional conversion between general CAD systems and Monte Carlo simulation codes (e.g. MCNP and TRIPOLI). The current version has already been developed into a practical program with all the necessary functions. The applications to the ITER building model, FFHR model and other large-scale nuclear devices have demonstrated the feasibility, effectiveness and maturity of MCAM latest version for nuclear applications with complex geometry.

MCAM is also a sub-system of the multi-functional integrated $4 \mathrm{D}$ neutronics simulation system VisualBUS ${ }^{15}$ ) developed by FDS Team. MCAM, as well as other professional codes ${ }^{16)}$ developed by FDS Team to be proposed as an exercise of development of nuclear informatics, is a necessary tool for advanced nuclear system design and analysis.

Due to the generic approach of MCAM, it is not limited to the specific Monte Carlo codes MCNP and TRIPOLI, but it can be easily extended to support other Monte Carlo codes in the future.

\section{Acknowledgments}

This work was supported by the National Natural Science Foundation of China with the grant Nos.10675123, 10875145, 10872212, and 50676108 and was also partly supported by the JSPS-CAS Core-University Program in the field of "Plasma and Nuclear Fusion".

\section{References}

1) J. F. Briesmeister (Ed.), MCNP - A general Monte Carlo $N$-Particle transport code, version 4C, LA-13709-M, Los Alamos National Laboratory (LANL) (2000).

2) C. M. Diop, E. Dumonteil, F. X. Hugot et al., "An overview on the Monte Carlo particle transport code TRIPOLI-4," Trans. Am. Nucl. Soc., 97, 694-695 (2007).

3) P. P. P. Wilson, R. Feder, U. Fischer, M. Loughlin, L. Petrizzi, Y. $\mathrm{Wu}, \mathrm{M}$. Youssef, "State-of-the-art 3-D radiation transport methods for fusion energy systems," Fusion Eng. Des., 83[7-9], 824-833 (2008).

4) Y. Wu, FDS Team, "CAD-based interface programs for fusion neutron transport simulation," Fusion Eng. Des., 84[7-11], 1987-1992 (2009).

5) Y. Li, L. Lu, A. Ding, H. Hu, Q. Zeng, S. Zheng, Y. Wu, "Benchmarking of MCAM 4.0 with the ITER 3D model," Fusion Eng. Des., 82[15-24], 2861-2866 (2007).

6) Y. Wu, Y. Li, L. Lu, A. Ding, "Research and development of the automatic modeling system for Monte Carlo particle transport simulation," Chinese J. Nucl. Sci. Eng., 26[1], 20-27 (2006).

7) L. Lu, Y. K. Lee, J. Zhang, Y. Li, Q. Zeng, Y. Wu, "Development of Monte Carlo automatic modeling functions of MCAM for TRIPOLI-ITER application," Nucl. Instr. Meth. Phys. Res. $A, \mathbf{6 0 5}[3]$, 384-387 (2009).

8) Q. Zeng, L. Lu, A. Ding, Y. Li, H. Hu, S. Zheng, Q. Huang, Y. Chen, Y. Wu, H. Iida, "Update of ITER 3D basic neutronics model with MCAM," Fusion Eng. Des., 81, 2773-2778 (2006).

9) S. Zheng, M. Chen, J. Li, Q. Zeng, L. Lu, Y. Li, A. Ding, "Neutronics analysis for the test blanket modules proposed for EAST and ITER," Nucl. Fusion, 47, 1053-1056 (2007).

10) $\mathrm{Y}$. $\mathrm{Wu}$, "Conceptual design activities of FDS series fusion power plants in China," Fusion Eng. Des., 81, 2713-2718 (2006).

11) Q. Huang, S. Zheng, L. Lu, "Neutronics analysis for a compact reversed shear tokamak CREST," Fusion Eng. Des., 81[8-14], 1239-1244 (2006).

12) A. Sagaraa, O. Mitaraib, S. Imagawaa, T. Morisakia, "Conceptual design activities and key issues on LHD-type reactor FFHR," Fusion Eng. Des., 81[23-24], 2703-2712 (2006).

13) P. Batistoni, L. Petrizzi, U. Fisher, A. Serikov, K. Seidel, R. Villari, L. Auditore, "Validation of FENDL-2.1 nuclear library for use in ITER nuclear analysis," Proc. International Conference on Nuclear Data for Science and Technology, Apr. 22-27, 2007, Nice, France, 979-982 (2008). 
14) T. Tanaka, A. Sagara, T. Muroga, M. Z. Youssef, "Development of three-dimensional neutronics calculation system for design studies on helical reactor FFHR," Fusion Eng. Des., 81[23-24], 2761-2766 (2006).

15) Y. Wu, J. Li, Y. Li, Q. Zeng, M. Chen, S. Zheng, "An integrated multi-functional neutronics calculation and analysis code system: VisualBUS," Chinese J. Nucl. Sci. Eng., 27[4], 365-373
(2007).

16) Y. Wu, L. Hu, P. Long, Y. Luo, Y. Li, Q. Zeng, L. Lu, J. Zhang, J. Zou, D. Xu, Y. Bai, T. Zhou, h. Chen, L. Peng, Y. Song, Q. Huang, "Development of design and analysis software for advanced nuclear systems," Chinese J. Nucl. Sci. Eng., 30[1], 55-64 (2010). 